\title{
Molecular Cloning of Silver Carp and Bighead Carp Prolactin
}

\author{
Y. S. ChANG, ${ }^{*} \dagger$ F. L. HUANG, ${ }^{*} \stackrel{+}{\dagger}$ AND T. B. Lo ${ }^{*, \dagger}$ \\ *Institute of Biological Chemistry, Academia Sinica; $†$ Graduate Institute of Biochemical Sciences, and \\ $\ddagger$ Department of Zoology, National Taiwan University, P.O. Box 23-106, Taipei, Taiwan
}

Accepted December 24, 1991

\begin{abstract}
The cDNAs encoding the prolactin of silver carp (scPRL) and bighead carp (bcPRL) have been cloned. Deduced from the nucleotide sequences, both scPRL and bcPRL are composed of 187 amino acid residues. Only one residue is different between scPRL and bcPRL. Homology analysis indicates that scPRL and bcPRL are highly homologous to carp PRL (97\%), relatively conserved in relation to PRLs of salmon, trout, and tilapia (64-69\%), and diversified from avian and mammalian PRL (30-35\%). Similar to PRLs of other species of fish, scPRL and bcPRL lack the first $12 \mathrm{~N}$-terminal residues of avian and mammalian PRLs. 1992 Academic Press, Inc.
\end{abstract}

Prolactin (PRL) is a polypeptide hormone secreted from the pituitary gland. Together with growth hormone and placental somatomammotropin, it belongs to a family of hormones evolved from a common ancestral gene (Miller and Eberhardt, 1983; Nicoll et al., 1986). This family has been extended due to the discovery of mouse proliferin (Linzer and Nathans, 1984; Linzer et al., 1985), bovine PRL-related cDNA I (Schuler and Hurley, 1987), rat PRL-like protein A (Deb et al., 1989), and somatolactins of flounder, Atlantic cod, and chum salmon (Ono et al., 1990; Rand-Weaver et al., 1991; Takayama et al., 1991). In fish, PRL is involved in many biological functions such as osmoregulation, reproduction, behavior, and metabolism (Clarke and Bern, 1980).

The amino acid sequence of PRL had been determined chemically or deduced from the nucleotide sequence of cDNA in many species of vertebrates including human (Cooke et al., 1981), cattle (Miller et al., 1981), sheep (Li et al., 1967; Varma et al., 1989), pig (Li, 1976), rat (Cooke et al., 1980), mouse (Linzer and Talamantes, 1985), whale (Tsubokawa et al., 1985), chicken (Hanks et al., 1989), carp (Yasuda et al., 1987; Chao et al., 1988), salmon (Yasuda et al., 1986; Song et al., 1988), rainbow trout (Mercier et al., 1989), and tilapia (Yamaguchi et al., 1988; Rentier-Delrue et al., 1989). In order to obtain more information about the evolution of PRL, we determined the primary structures of PRL of silver carp (scPRL) and bighead carp (bcPRL) by using cDNA cloning technique. In this paper, the cDNA nucleotide sequences and the deduced amino acid sequences of scPRL and bcPRL are presented and their sequences are compared to those of PRLs from other fish and vertebrates.

\section{MATERIALS AND METHODS}

Construction of cDNA library and screening of $P R L C D N A$. The method used to construct cDNA library was essentially the same as previously described (Chang et al., 1990). Briefly, the pituitary polyadenylated RNAs of silver carp (Hypophthalamichthys molitrix) and bighead carp (H. nobilis) were prepared from liquid nitrogen frozen tissues by guanidinium/CsCl method (Ullrich et al., 1977) followed by oligo(dT)-cellulose column chromatography. The doublestranded cDNA, synthesized by the method of Gubler and Hoffman (1983), was ligated with EcoRI linker and subsequently inserted into EcoRI site of $\lambda$ gt10. To 
screen the recombinant. DNA clones containing the PRL cDNA from the cDNA libraries, the cDNA coding for carp PRL (CPRL) previously cloned in our laboratory was ${ }^{32} \mathrm{P}$-labeled by nick-translation and used as a hybridization probe. Plaque hybridization was performed according to the method of Benton and Davis (1977).
Nucleotide sequencing of $C D N A$ s encoding scPRL and $b c P R L$. The $\mathrm{CDNAs}$ encoding $\mathrm{SCPRL}$ and $\mathrm{bcPRL}$ were cleaved with Avall for subcloning. The nucleotide sequences were determined by the dideoxy chain termination method (Sanger et al., 1977) using supercoiled plasmid DNA as template (Chen and Seeburg, 1985).

GGCAAGAGAATAAAGGAACCAGTTAAAATAATGGCTGAAGGATCCAGACTATACTTTGCA

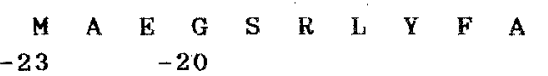

GTGACTGTCCTGATGTGTGCGTTTGTCTCAATCAACGGTGTCGGTCTGAATGATTTACTG 120

$\begin{array}{llllllllllllllllllll}\text { V } & \text { T } & \text { V } & \text { L } & \text { M } & \text { C } & \text { A } & \text { F } & \text { V } & \text { S } & \text { I } & \text { N } & \text { G } & \text { V } & \text { G } & \text { L } & \text { N } & \text { D } & \text { ls } & \text { L }\end{array}$

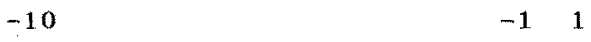

GAAAGAGCCTCTCAACTTTCAGACAAACTTCACTCCCTCAGCACCTCTCTCACCAATGAC 180

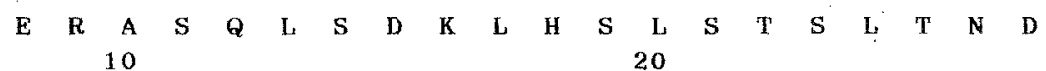

CTGGATTCTCACTTTCCTCCTGTTGGGAGGGTAATGATGCCCCGTCCGTCGATGTGCCAC 240

$\begin{array}{llllllllllllllllllll}\mathbf{L} & \mathbf{D} & \mathbf{S} & \mathbf{H} & \mathbf{F} & \mathrm{P} & \mathbf{P} & \mathbf{V} & \mathbf{G} & \mathbf{R} & \mathrm{V} & \mathbf{M} & \mathbf{M} & \mathbf{P} & \mathrm{R} & \mathrm{P} & \mathrm{S} & \mathbf{M} & \mathrm{G} & \mathrm{H}\end{array}$ 30

ACATCCTCCCTRCAAATTCCCAATGACAAAGACCAAGCCCTGAAAGTGCGGGAGGATGAG 300 $\begin{array}{llrlllllllllllllllll}\text { T } & \text { S } & \begin{aligned} \text { S } \\ \text { L }\end{aligned} & \text { L } & \text { Q } & \text { I } & \text { P } & \text { N } & \text { D } & \text { K } & \text { D } & \text { Q } & \mathbf{A} & \text { L } & \text { K } & \text { V } & \text { P } & \text { E } & \text { D } & \text { E }\end{array}$

TTACTT TCTTTGGCTCGGTCTCTGCTGCTGGCGTGGTCCGATCCCCTGGCCCTCCTCTCC 360

$\begin{array}{lllllllllllllllllllll}\text { L } & \text { L } & \text { S } & \text { L } & \text { A } & \text { R } & \text { S } & \text { L } & \text { L } & \text { L } & \text { A } & \text { W } & \text { S } & \text { D } & \text { P } & \text { L } & \text { A } & \text { L } & \text { L } & \text { S }\end{array}$ $70 \quad 80$

TGTGAGGCGTCCAGCCTGGGACATCCAGAACGCAACACCATTAACAGCAAGACCAAAGAA 420

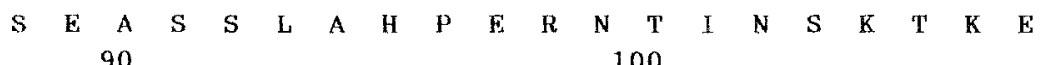

CTGCAAGACAACATCAACAGCCTGGG'TGCAGGTCTGGAGCGTGTCGTTCACAAGATGGGC 480

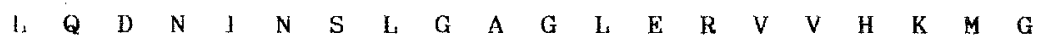
110

ICATCCTCAGACAACCTGTCCTCTCTCCCTTTTTACAGCAACAGCCTTGGCCAGGATAAA 540

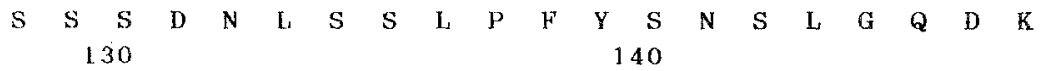
ACC'TCTCGACTTGTCAATTTCCATTTTCTG'TTTCCTGCTTCCGCAGGGACTCCCACAAA 600

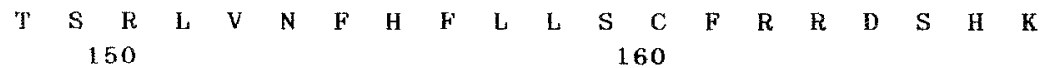
ATI'GACAGTTTCCTCAAAGTTCTGCGCTGCCGGGCAGCGAAGAAGAGACCTGAGATGTGC 660 $\begin{array}{llrlllllllllllllllll}1 & D & S & F & L & K & V & L & K & C & R & A & A & K & K & R & P & E & M & C \\ 170 & & & & & & & & & 180 & & & & & & & 187\end{array}$ TAAAGTGAAATGCTACTCTGCTTCTCTCATTGTGGATGTTAGGTTAAAATGGCAGAGCA 720

GTGAGTGATTTGAAATGTTTCTTATAATACCGCATGGCAAAACATGCCCTTTATTGTTT 780

CAAAGATGTAGA'TATTTGATTCACTTTCTTATATACTTGAATAAATAACTGGACCAAGGG 840

GCATATTATTCTGTCCCCAGAGATAAAATACAACAAAAAGTAAACAAAAGTATTTCAATT 900

AAATT"TAAAATRGAAGGTCATATAAAAGGTCTGCATGGAGTCATACTGTTTGATGAATA 960

AATGTATATTGAAATGTTTGACCATGCTCAGTTTAATAAGACATTTGTATAGTAAGTATT 1020

TATTAAGAAATGATGTTGGTCTTTAACACGTTTATTTTAACATTATCTTCTGAAATGCA 1080

FIG. 1. The nucleotide sequence and deduced amino acid sequence of bcPRL cDNA. 


\section{RESULTS AND DISCUSSION}

When cPRL cDNA was used as a probe, 8 and 9 positive clones were obtained from 5000 and 6000 clones from silver carp and bighead carp pituitary cDNA library, respectively. In this investigation, only the clone with the largest insert of each library was chosen for further study.

The cDNA encoding bcPRL investigated in this study is $1170 \mathrm{bp}$ in length, consisting of a $5^{\prime}$-untranslated region of $30 \mathrm{bp}$, an open reading frame $(\mathrm{ORF})$ of $630 \mathrm{bp}$, and a $3^{\prime}$ untranslated region of $510 \mathrm{bp}$. The ORF encodes a signal peptide of 23 amino acid residues and a putative mature PRL of 187 amino acid residues. Its nucleotide sequence and deduced amino acid sequence are presented in Fig. 1.

The cDNA encoding scPRL investigated in this study is $1060 \mathrm{bp}$ in length. The nucleotide sequence of cDNA encoding ScPRL is highly homologous to that of cDNA encoding bcPRL (data not presented). It contains an ORF of $621 \mathrm{bp}$ and a 3 '-untranslated region of $436 \mathrm{bp}$. The ScPRL cDNA thus obtained is not full in length, lacking the translation initiation codon, AUG, and the poly(A) tract. Among the 207 amino acid residues coded by ORF, a putative mature PRL of 187 residues and an incomplete signal peptide of 20 residues are present.

The scPRL and bcPRL are similar to each other. Only one of 187 amino acid residues is changed (Fig. 2). When scPRL and bcPRL were compared to cPRL, from another species of Cyprinidae, a high extent of homology (97\%) was found (Yasuda et al., 1987; Chao et al., 1988). A similar situation was also found among the PRLs of three species of Salmonidae: chum salmon, chinook salmon, and rainbow trout where the homology of PRL is $98 \%$ or more (Yasuda et al., 1986; Song et al., 1988; Mercier et al., 1989). On the contrary, when PRLs of different fish families were compared (Fig. 2), homology is decreased to the range of 64 to $74 \%$ (Cyprinidae vs Salmonidae, 69\%; Cyprinidae vs tilapia, 64\%; Salmonidae vs tilapia, 74\%) (Yasuda et al., 1986, 1987; Song et al., 1988; Yamaguchi et al., 1988; Mercier et al., 1989; Rentier-Delrue et al., 1989).

Most fish PRLs thus far investigated are composed of 187 to 189 amino acid residues except 177 residues are found in one form of two tilapia PRLs (Yamaguchi et al., 1988; Rentier-Delrue et al., 1989). In comparison, chicken PRL has 198 residues while ovine and human PRL have 199 residues. Such difference arises from the absence in fish PRLs of the first $12 \mathrm{~N}$-terminal residues of higher vertebrates. Homology between fish and higher vertebrate PRL is low, about 30 to $35 \%$ (Li et al., 1967; Li, 1976; Cooke et al., 1980, 1981; Miller et al., 1981; Linzer and Talamantes, 1985; Tsubokawa et al., 1985; Hanks et al., 1989; Varma et al., 1989). Although the structure of PRLs of fish and higher vertebrates are diversified, two conserved segments of almost identical or chemically similar sequence, residues 46 to 51 and 160 to 180 , were found in all PRLs thus investigated. These conserved sequences may be important for the biological activity of PRL. As shown in Fig. 2, PRLs of fish contain four cysteine residues while those of higher vertebrates contain six cysteine residues except for equine PRL ( $\mathrm{Li}$ and Chung, 1983). The first two cysteine residues of higher

FIG. 2. Comparison of the primary structure of scPRL and bcPRL with PRLs of other fish and higher vertebrates. Residues identical to scPRL are indicated by dashes. Gaps, marked by X, are inserted to maximize structural alignment. References used: carp, Yasuda et al. (1987); chum salmon, Yasuda et al. (1986); chinook salmon, Song et al. (1988); rainbow trout, Mercier et al. (1989); $O$. mossambicus I and II, Yamaguchi et al. (1988); O. nilotica I and II, Rentier-Delrue et al. (1989); chicken, Hanks et al. (1989); sheep, Varma et al. (1989); human, Cooke et al. (1981). 
Silver carp (1)

Bighead carp (2)

Carp (3)

Chinook salmon (5)

Rainbow lrout (6)

o. mossambicus I (7)

o. niloticus I (8)

o. mossambicus II (9)

o. niloticus II (10)

Chicken (11)

sheep (12)

Human (13)
Chum salmon (4)

1

10

20

30

40

VGLNDLLERASQLSDKLHSLSTSLTNDLDSHFPPVGRVMM

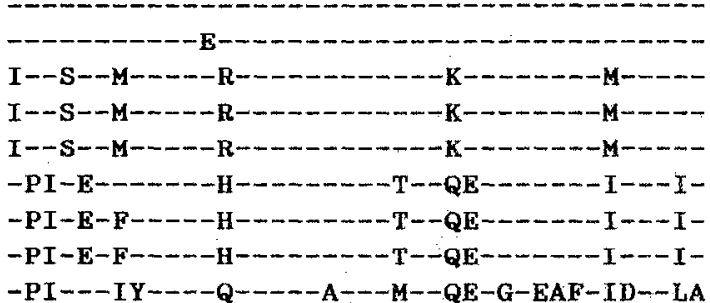

LPICPIGSVNCQ-S-GE-FD-VK--HYI-Y--SEIF-EF-ERYAQGRGF IT IPVCPNGPGDCQ-S-R-DD--VMV-HYI-N-DEMF-EF-KRYAQGKGF IT LPICPGGAARCQ-T-R--FD-VV--HYI-N-SEMFSEF-KRYTHGRGFIT

50

60

70

80

90

100

1. PRPSMCHTSSLQTPNDKDQALKVPEDELLSLARSLLLAWSDPLALLSSEASSLAHXPERNT

2 .

3 .

4.

5.

6.

7 .

8.

9.

10.

11 .

12 .

13.

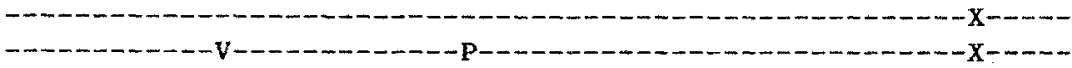

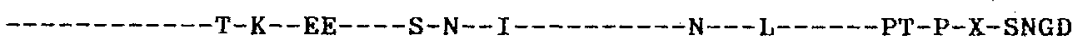

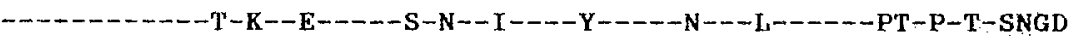

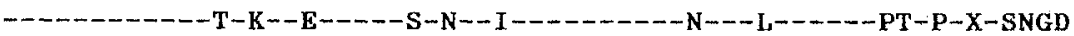

$--\mathrm{A}-----\mathrm{T}-\mathrm{I}----\mathrm{Q}-\mathrm{S}-\mathrm{SD}-\mathrm{M}-\cdots---\mathrm{Q}-\cdots-\cdots-\mathrm{VV}-\mathrm{-}-\mathrm{S}-\mathrm{T}-\mathrm{P}-\mathrm{X}-\mathrm{AQSS}$

$---\mathrm{A}--\cdots-\mathrm{T}-\mathrm{I}-\cdots---\mathrm{Q}-\mathrm{S}-\mathrm{SD}-\mathrm{M}--\cdots---\mathrm{Q}-----\mathrm{VV}-\cdots-\mathrm{S}-\mathrm{T}-\mathrm{P}-\mathrm{X}-\mathrm{AQS}-$

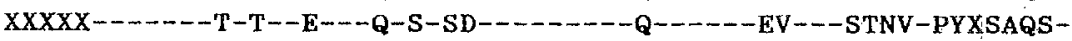

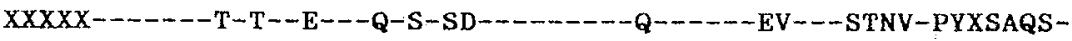

KAVNG-- - - TT-E--E--QQRHHED--N-VVGV-RS-N--DIH-A- VQRIKEA-DTIL

MALNS-- - - PT-E--E--QQTHHEV-M- I LG--RS-N-- YH-VT-VRGMKGV-DAIL

KAINS-- - -AT-E--E-QQRNQKDF-- IV-I-RS-NE--YH-VT-VRGMQEA--AII

110

120

130

140

150

160

1. INSKTKELQDN INSLGAGLEHVVHKMGSSSDNLSSLPFYSNSLXGQDKTSXRLVNFHFLLSC

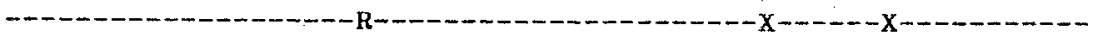

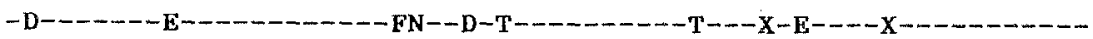
$-\mathrm{S}--\mathrm{IR}-\cdots \mathrm{YSK}-\cdots-\mathrm{D}--\mathrm{DIM}-\mathrm{N}---\mathrm{P}--\mathrm{QY} \mathrm{I}--\mathrm{I}--\mathrm{KGGD}-\mathrm{X}-\mathrm{N}----\mathrm{X}--\mathrm{I}--\mathrm{M}-\mathrm{M}--$

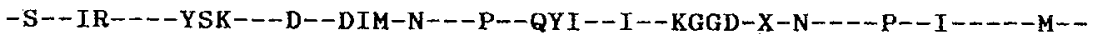
$-\mathrm{S}--\mathrm{IR}----\mathrm{YSK}-\cdots-\mathrm{D}--\mathrm{DIM}-\mathrm{N}---\mathrm{P}--\mathrm{QY} \mathrm{I}--\mathrm{I}--\mathrm{KGGD}-\mathrm{X}-\mathrm{N}----\mathrm{X}--\mathrm{I}-\cdots-\cdots-\mathrm{M}--$ -FN-IQ-M-QYSK--KD--DVLSS----PAQAIT---YRGGTNL-H--ITXK-I--N-----FN-IQ-M-QYSK--KD--DVLSS----PAQATT-- YRGGTNL-H-ITXK-I--N----LSKT IQKM-EHSKD-KD--DI LSS---PAAQTIT-- - IETNET--- ITXKXXXXXX--LSKTIQKM-EHSKD-KD--DILSS-- PAAQTIT--- IETNET----ITXKXXXXXX---WKAVEI-E-NKRLLE-MEKIVGRVHS-DAGNEIY-HWDGLP--QLA-ED-X--FA-YN-DSRAIEI-EENKR----MEMIFGEVIP-AKETEPYPVWSGLP-QTK-EDAX-HSA-YN--HSKAVE I - E-TKRLLE-ME-IVSQVHPETKENETYPVWSGLP--QHA-EE-X--SAYYN--H-

170 $180 \quad 187$

1.

FRRDSHKIDSFLKVLRCRAAKKRPEMC

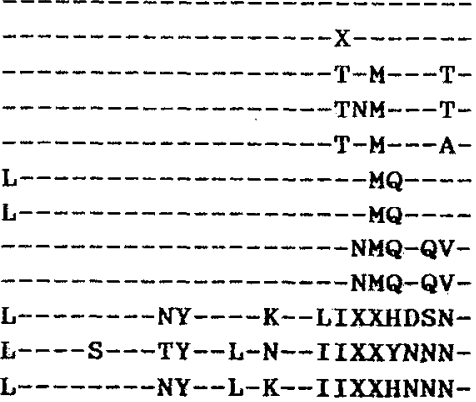


vertebrate PRLs are located in the segment of the first $12 \mathrm{~N}$-terminal residues which are lacking in fish PRLs. All the four cysteine residues of fish PRLs can be aligned at the same positions of PRLs of higher vertebrates. In salmon PRL, two disulfide linkages are also formed between the corresponding cysteine residues of mammalian PRLs, i.e., $\mathrm{Cys}_{46-160}$ and $\mathrm{Cys}_{177-187}$ (Yasuda et al., 1986). Whether such disulfide linkages are present in cyprinid PRLs remains to be studied.

\section{ACKNOWLEDGMENT}

This work was in part supported by the National Science Council, Republic of China.

\section{REFERENCES}

Benton, W. D., and Davis, R. W. (1977). Screening $\lambda$ gt recombinant clones by hybridization to single plaque in situ. Science 196, 180-182.

Chang, Y. S., Huang, C. J., Huang, F. L., Liu, C. S., and Lo, T. B. (1990). Purification, characterization, and molecular cloning of gonadotropin subunits of silver carp (Hypophthalmichthys molitrix). Gen. Comp. Endocrinol. 78, 23-33.

Chao, S. C., Pan, F. M., and Chang, W. C. (1988). Nucleotide sequence of carp prolactin cDNA. Nucleic Acids Res. 16, 9350.

Chen, E. J., and Seeburg, P. H. (1985). Supercoiled sequencing: A fast and simple method for sequencing plasmid DNA. DNA 4, 165-170.

Clarke, W. C., and Bern, H. A. (1980). Comparative endocrinology of prolactin. In "Hormonal Proteins and Peptides" (C. H. Li, Ed.), pp. 105-197. Academic Press, New York.

Cooke, N. E., Coit, D., Weiner, R. I., Baxter, J. D., and Martial, J. A. (1980). Structure of cloned DNA complementary to rat prolactin messenger RNA. J. Biol. Chem. 255, 6502-6510.

Cooke, N. E., Coit, D., Shine, J., Baxter, J. D., and Martial, J. A. (1981). Human prolactin cDNA structural analysis and evolutionary comparisons. J. Biol. Chem. 256, 4006-4016.

Deb, S., Youngblood, T., Rawitch, A. B., and Soares, M. J. (1989). Placental prolactin-like protein A: Identification and characterization of two major glycoprotein species with antipeptide antibodies. J. Biol. Chem. 264, 14,348-14,353.

Gubler, U., and Hoffman, B. J. (1983). A simple and very efficient method for generating cDNA library. Gene 25, 263-269.
Hanks, M. C., Alonzi, J. A., Sharp, P. J., and Sang, H. M. (1989). Molecular cloning and sequence analysis of putative chicken prolactin cDNA. $J$. Mol. Endocrinol. 2, 21-30.

Li, C. H. (1976). Studies on pituitary lactogenic hormone. The primary structure of the porcine hormone. Int. J. Pept. Protein Res. 8, 205-224.

Li, C. H., Dixon, J. S., Lo, T. B., Pankow, Y. M., and Schmidt, K. D. (1967). Amino acid sequence of ovine lactogenic hormone. Nature 224, 695696.

Li, C. H., and Chung, D. (1983). Studies on prolactin 48: Isolation and properties of the hormone from horse pituitary glands. Arch. Biochem. Biophys. 220, 208-213.

Linzer, D. I. H., and Nathans, D. (1984). Nucleotide sequence of a growth-related mRNA encoding a member of the prolactin-growth hormone family. Proc. Natl. Acad. Sci. USA 81, 4255-4259.

Linzer, D. I. H., and Talamantes, F. (1985). Nucleotide sequence of mouse prolactin and growth hormone mRNAs and expression of these mRNAs during pregnancy. J. Biol. Chem. 17, 9574-9579.

Linzer, D. I. H., Lee, S. J., Ogren, L., Talamantes, F., and Nathans, D. (1985). Identification of proliferin mRNA and protein in mouse placenta. Proc. Natl. Acad. Sci. USA 82, 4356-4359.

Mercier, L., Rentier-Delrue, F., Swennen, D., Lion, M., Le Coff, P., Prunet, P., and Martial, J. A. (1989). Rainbow trout prolactin cDNA cloning in Escherichia coli. DNA 8, 119-125.

Miller, W. L., Coit, D., Baxter, J. D., and Martial, J. A. (1981). Cloning of bovine prolactin cDNA and evolutionary implications of its sequence. DNA 1, 37-50.

Miller, W. L., and Eberhardt, N. L. (1983). Structure and evolution of the growth hormone family. Endocrinol. Rev. 4, 97-130.

Nicoll, C. S., Mayer, G. L., and Russell, S. M. (1986). Structural features of prolactins and growth hormones that can be related to their biological properties. Endocrinol. Rev. 7, 169-203.

Ono, M., Takayama, Y., Rand-Weaver, M., Sakata, S., Yasunga, T., Noso, T., and Kawauchi, H. (1990). cDNA cloning of somatolactin, a pituitary protein related to growth hormone and prolactin. Proc. Natl. Acad. Sci. USA 87, 4330-4334.

Rand-Weaver, M., Noso, T., Muramoto, K., and Kawauchi, H. (1991). Isolation and characterization of somatolactin, a new protein related to growth hormone and prolactin from Atlantic cod (Gadus morhua) pituitary glands. Biochemistry 30, 1509-1515.

Rentier-Delrue, F., Swennen, D., Prunet, P., Lion, M., and Martial, J. A. (1989). Tilapia prolactin: 
Molecular cloning of two cDNAs and expression in Escherichia coli. DNA 8, 261-270.

Sanger, F., Nicklen, S., and Coulson, A. R. (1977). DNA sequencing with chain-terminating inhibitors. Proc. Natl. Acad. Sci. USA 74, 5463-5467.

Schuler, L. A., and Hurley, W. L. (1987). Molecular cloning of a prolactin-related mRNA expressed in bovine placenta. Proc. Natl. Acad. Sci. USA 84, $5650-5654$.

Song, S., Trinh, K. Y., Hew, C. L., Hwang, S. J., Belkhode, S., and Idler, D. R. (1988). Molecular cloning and expression of salmon prolactin cDNA. Eur, J. Biochem. 172, 279-285.

Takayama, Y., Rand-Weaver, M., Kawauchi, H., and Ono, M. (1991). Gene structure of chum salmon somatolactin, a presumed pituitary hormone of the growth hormone/prolactin family. Mol. Endocrinol. 5, 778-786.

Tsubokawa, M., Muramoto, K., and Kawauchi, H. (1985). Primary structure of fin whale prolactin. Int. J. Pept. Protein Res. 25, 242-248.
Ullrich, A., Shine, J., Chirgwin, J., Pictet, R., Tischer, E., Rutter, W. J., and Goodman, H. M. (1977). Rat insulin genes: Construction of plasmid containing the coding sequences. Science 196, 1313-1319.

Varma, S., Kwik, S, and Ebner, K. E. (1989), Cloning and nucleotide sequence of ovine prolactin cDNA. Gene 77, 349-359.

Yamaguchi, K., Specker, J. L., King, D. S., Yokoo, Y., Nishioka, R. S., Hirano, T., and Bern, H. A. (1988). Complete amino acid sequences of a pair of fish (Tilapia) prolactins, tPRL $_{177}$ and $\mathrm{TPRL}_{188}$. J. Biol. Chem. 263, 9113-9121.

Yasuda, A., Itoh, H., and Kawauchi, H. (1986); 1 rimary structure of chum salmon prolactins: Occurrence of highly conserved regions. Arch. Biochem. Biophys. 244, 528-541.

Yasuda, A., Miyazima, K. I., Kawauchi, H., Peter, R. E., Lin, H. R., Yamaguchi, K., and Sano, H. (1987). Primary structure of common carp prolactin. Gen. Comp. Endocrinol. 66, 280-290. 